\title{
Influence of Saturation of Magnetic Lens Material on Fields of Deflectors and Parasitic Fields
}

\author{
J. Zlámal ${ }^{1,2}$, B. Lencová ${ }^{1,3}$ \\ ${ }^{1 .}$ Institute of Physical Engineering, Brno University of Technology, Technická 2, 61669 Brno, Czech \\ Republic \\ 2. CEITEC BUT, Technická 10, 61669 Brno, Czech Republic \\ 3. TESCAN Brno, s.r.o., Libušina tř. 1, 62300 Brno, Czech Republic
}

\section{Introduction}

The program EOD [1] allows high accuracy computation of saturated magnetic lenses as well as parasitic fields due to machining and assembly tolerances, if the first order finite element method is used with sufficiently dense meshes with 100,000 points or more. The accuracy of magnetic lens computations is discussed in length in [2], the accuracy of deflection field computations in [3], and the computation algorithm is in [4].

In the connection with the calculation of parasitic fields of highly saturated lens in [5] emerged the problem of calculation of the field of deflectors situated next to saturated magnetic materials. Up to date the deflection fields were studied only near rotationally symmetric materials with constant permeability. However, for the deflectors in saturated magnetic lenses the relative permeability is very inhomogeneous and near pole piece tips it is close to 1 . We have therefore modified the computation of deflection fields in saturated lenses with EOD program [1] to include the real value of permeability of magnetic materials. We can also display the distribution of permeability in the magnetic circuit of the lens to understand the changes in the field distribution in deflection or parasitic fields and to understand the saturation effects in magnetic lenses.

\section{Deflection fields}

The only computation of deflectors taking into account the value of relative permeability of magnetic material was published by Scherle [7], see also [8], chapter 40. The computations of Scherle for toroidal coil on cylindrical magnetic former and saddle coil next to magnetic cylinder with constant relative permeability were repeated for several values of permeability. The results shown in Figure 1 are quite similar to that of [8]. The axial flux density for the toroidal coil (Figure 1a) is increased approximately four times if we place the coil on a magnetic former with high permeability compared to the field in air. The strength of axial dipole function of saddle coils (Figure 1b) is increased almost twice. Obviously, the effect of slightly different field shape is negligible, because the deflection angle and the aberrations of dipole field depend mainly on the effective field width [10].

More complicated situation arises if the deflection coils are situated in the vicinity of a saturated material. Permeability is no more homogeneous as in previous cases. As an example we studied a radial coil in the vicinity of the upper part of the left-hand pole piece of the $200 \mathrm{keV}$ saturated magnetic lens of Tsuno [6] shown in Figure 2, recently examined by Sháněl et al [5]. 


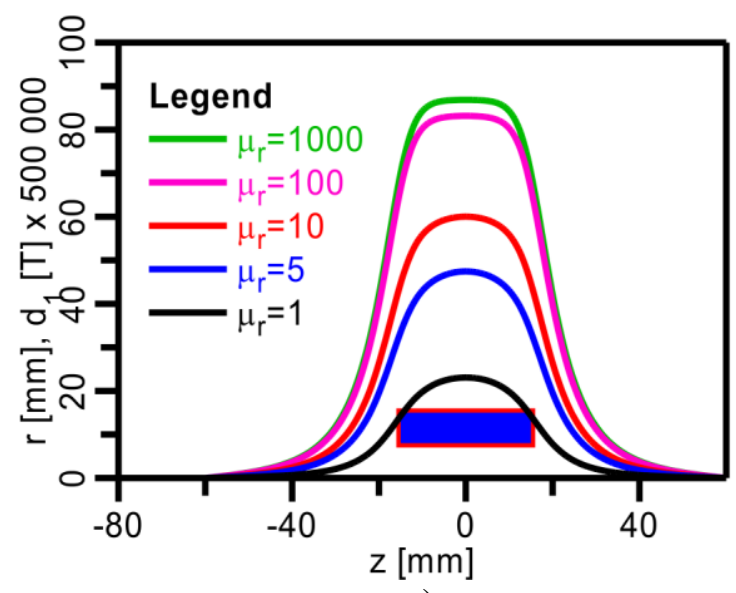

a)

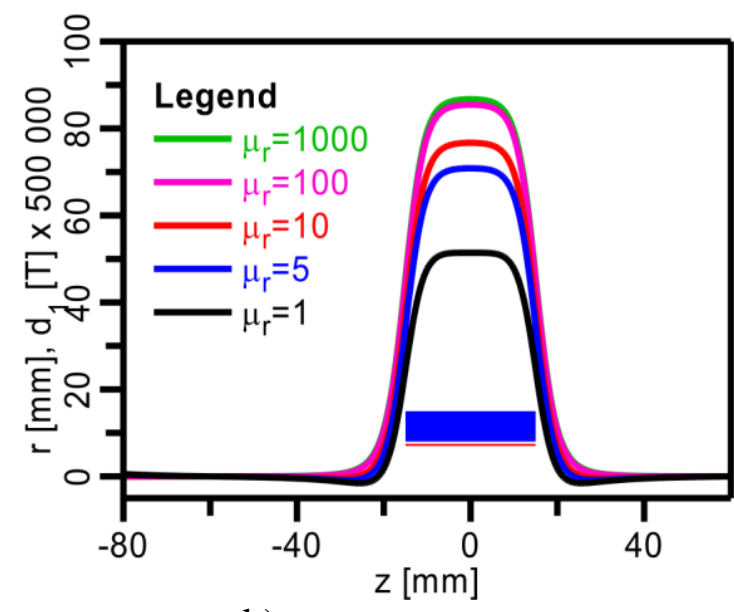

b)

Figure 1. Axial deflection field for the relative permeability of material ranging from 1 to 1000 . a) The toroidal coil on the magnetic cylinder. b) The saddle coil placed next to magnetic cylinder.

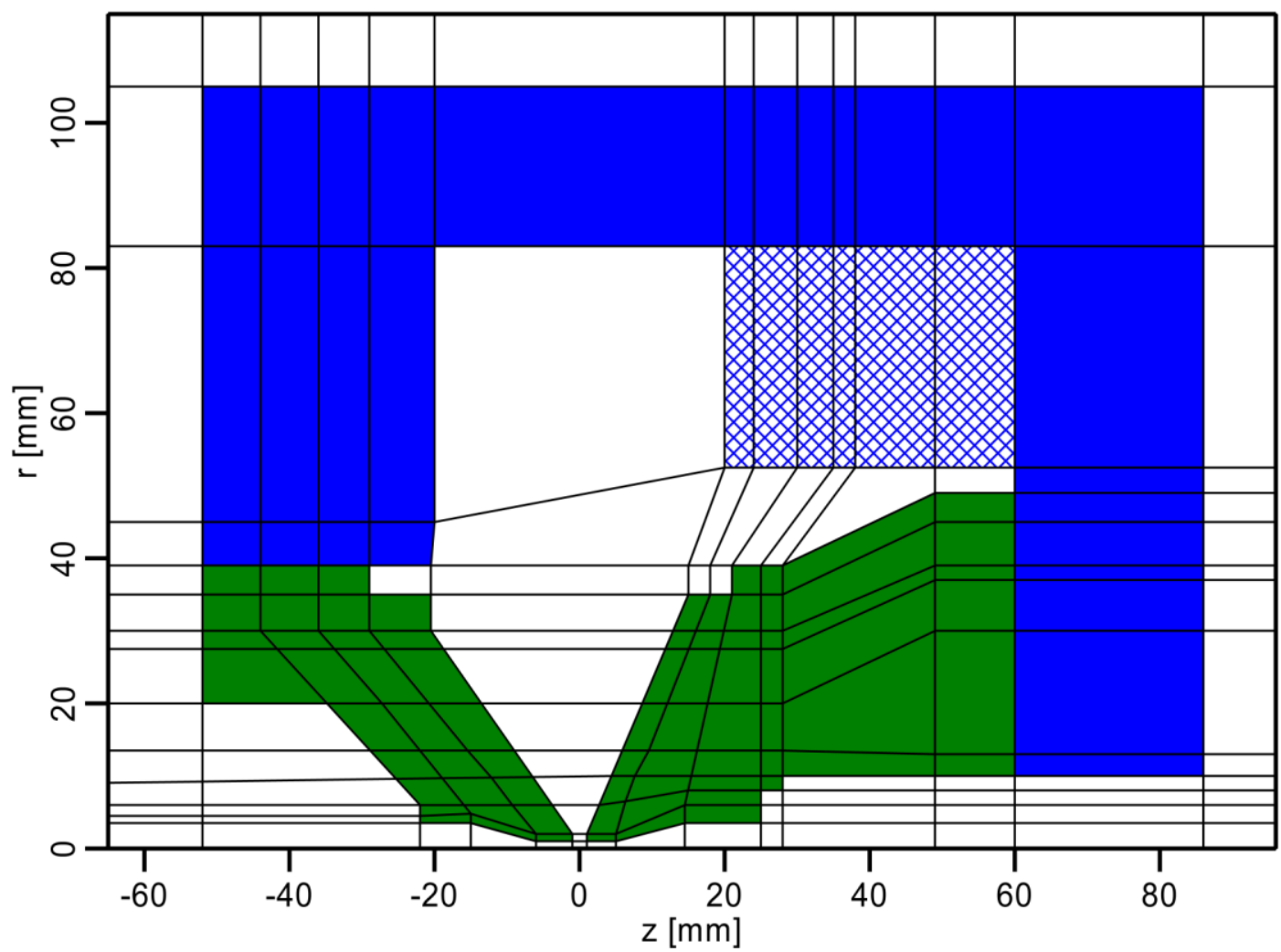

Figure 2. 200keV objective lens of Tsuno [6]. Lens gap and bore diameter are both $2 \mathrm{~mm}$.

The results presented in this paper are calculated in the mesh with 330000 mesh points and mesh step 1/60 $\mathrm{mm}$ in the gap for lens excitations 500 and 16000 Ampere-turns. Material of pole pieces remains linear (except for a small area near the pole piece tip) for the excitation 500 Ampere-turns, in contrast to 16000 Ampere-turns excitation when the material of the pole pieces is fully saturated (nonlinear). The 
radial coil, defined with the help of vertical coarse mesh lines, produces magnetic flux perpendicular to the material boundary and the field can spread into the magnetic material if the lens saturates.

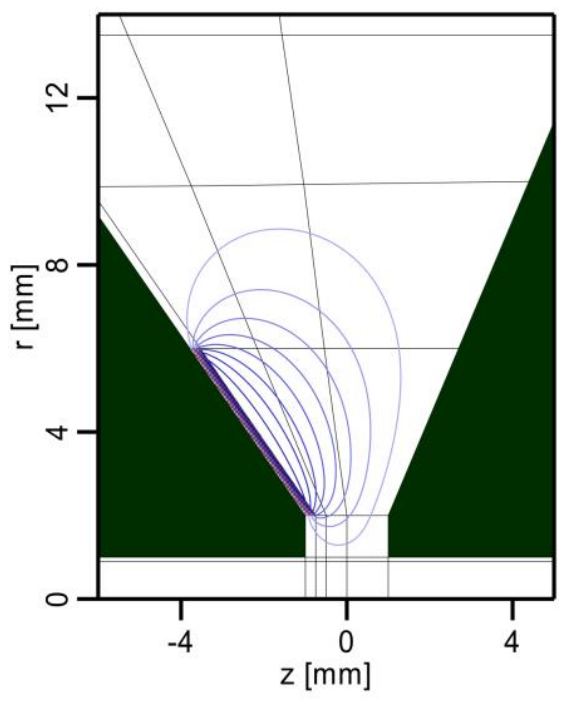

a)

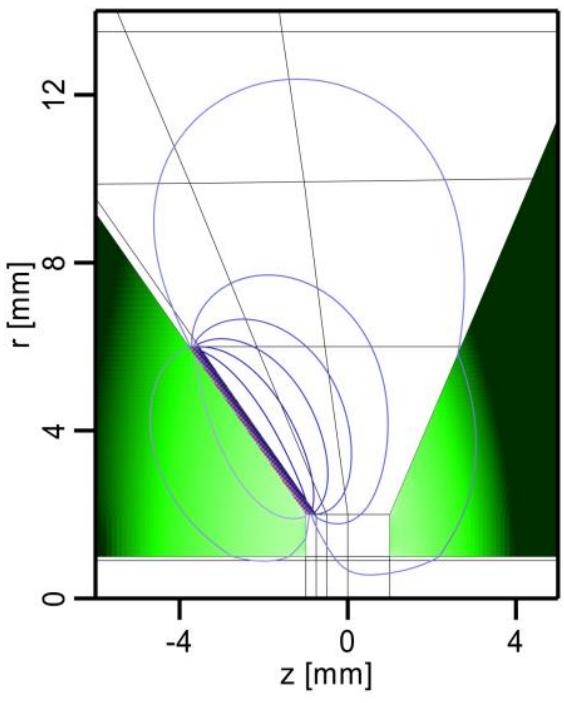

b)
Legend:

Permeability

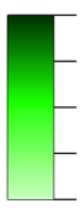

5.00

4.25

3.50

2.75

2.00

Figure 3. Equipotentials of the dipole component of the scalar magnetic potential of radial coil in the vicinity of the upper part of left-hand pole piece of $200 \mathrm{keV}$ lens of Tsuno. a) nonsaturated lens (linear case) - relative permeability of material is higher than 1000, b) saturated lens (nonlinear case) - relative permeability of material can be less than 5 .

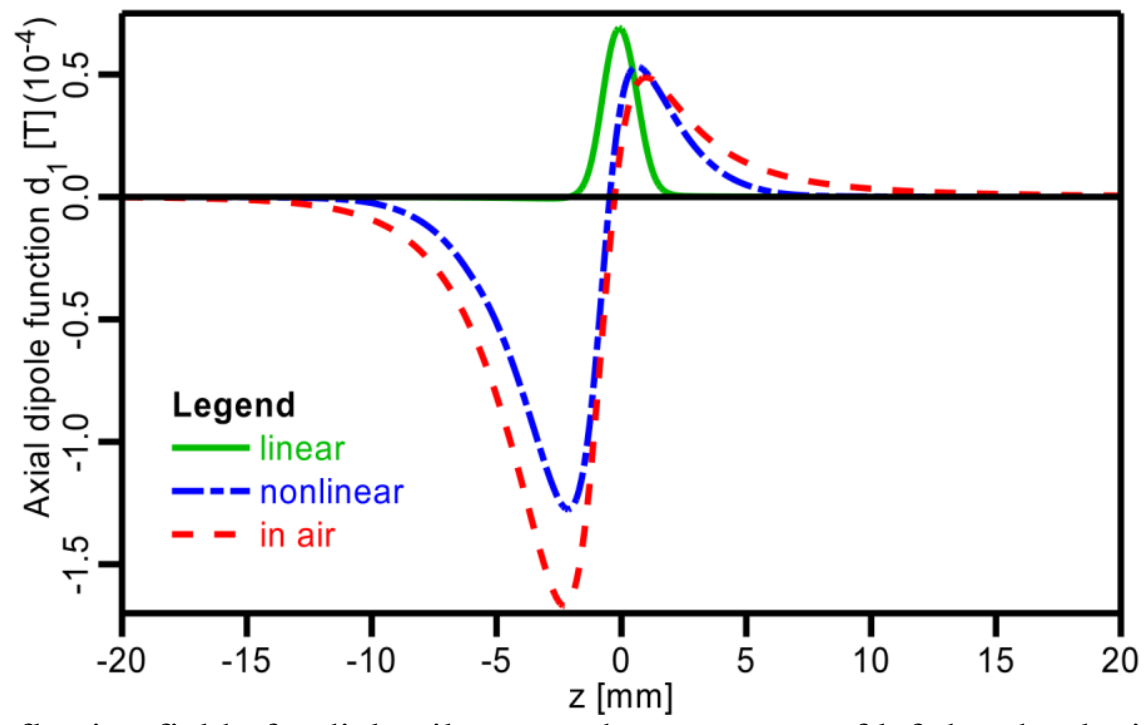

Figure 4. Axial deflection field of radial coil next to the upper part of left-hand polepiece.

The details of the pole piece region with the equipotentials of the dipole component of the scalar magnetic potential of the coil in the nonsaturated and saturated lens are shown in Figure 3. In the linear case is the field on the axis localized only in the lens gap (Figure 3a) because it can spread only in air. The low relative permeability of the saturated material (below 5 in polepiece region) cause easier penetration of the field to the area near the optical axis and into the opposite pole piece (Figure 3b). Axial deflection field of the coil is shown in Figure 4. Dipole field of linear case is present only in the 
lens gap. The deflection field of coil near saturated material (nonlinear case) is much higher under the upper pole piece because it can penetrate through the low permeability material towards the axis. This shape of the deflection field is easy to understand if we compare it with the field of the same coil placed only in air, with no magnetic material present, but in the same computation mesh. We can say that the magnetic material in the nonlinear case effectively almost disappears.

\section{Parasitic fields}

Parasitic fields of shift, tilt, and ellipticity can be calculated by perturbation theory proposed by Sturrock [9]. They have been utilized recently by Sháněl et al [5] using saturated $200 \mathrm{kV}$ lens of Tsuno [6]. In Sturrock's theory [9] it is explicitly stated that the surface of the magnetic potential is an equipotential of the magnetic scalar potential. We supposed that the theory is true even for saturated lenses for which this condition is not fulfilled. For the computation of parasitic fields, the field on the lens outside is used as a boundary condition for their computation, so it implicitly accounts for lens saturation. For small shift of a perfect pole piece by $s$ perpendicular to optical axis is the boundary condition for dipole component of scalar magnetic potential on perturbed material boundaries

$$
\Phi_{1}=-s m_{r} \vec{m} \cdot \vec{H}
$$

where $\vec{m}$ is outer normal of the perturbed boundary $\left(m_{r}\right.$ is radial component of $\left.\vec{m}\right)$ and $\vec{H}$ is magnetic field intensity. Similarly the boundary condition for the quadrupole component of scalar magnetic potential for ellipticity $\varepsilon$ is

$$
\Phi_{2}=-\varepsilon m_{r} r \vec{m} \cdot \vec{H}
$$

Tilt is for simplicity not considered. On the unperturbed boundaries is the boundary condition of parasitic fields zero.

Parasitic fields were evaluated using EOD and also by the 3D calculation in COMSOL Multiphysics to find the limits of the perturbation theory. COMSOL uses the second order finite element method in three dimensions to calculate magnetic vector potential of saturated magnetic lens. Because the shift and ellipticity can be quite small, we had to use quite dense and large meshes, e.g. mesh step $0.1 \mathrm{~mm}$ in the polepiece region. We could use up to 3 million elements, but the computation runs then about one day. The objective lens used before was studied for two different excitations, 500 and 16000 Ampere-turns. In the computation the perturbed lens part was the whole inner surface of the left hand pole piece.

At 500 Ampere-turns the lens is not yet saturated and the relative permeability of the material is high. Magnetic field lines for excitation 500 Ampere-turns are almost perpendicular to the perturbed surface, which correspond to the theory of Sturrock. The resulting parasitic fields calculated in EOD using the perturbation theory are in good agreement with the results obtained from 3D calculation performed in COMSOL Multiphysics software (Figure 5).

On the contrary, for the excitation of 16000 Ampere-turns the relative permeability of material is very low. The magnetic field lines are not perpendicular to the polepiece surface any more. The difference of the axial field functions calculated using perturbation theory in EOD and from 3D calculation is much bigger (Figure 6). The slanted part of the pole piece also starts to play a role. 


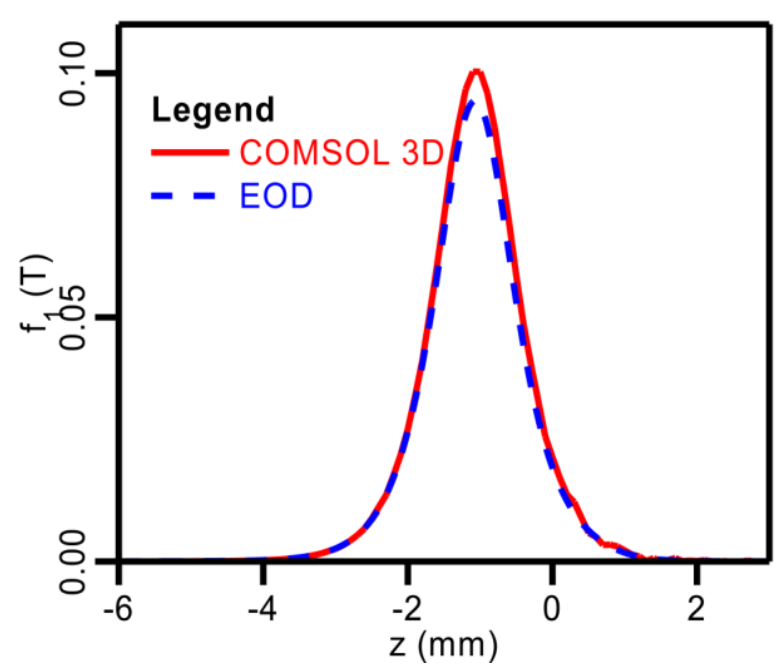

a)

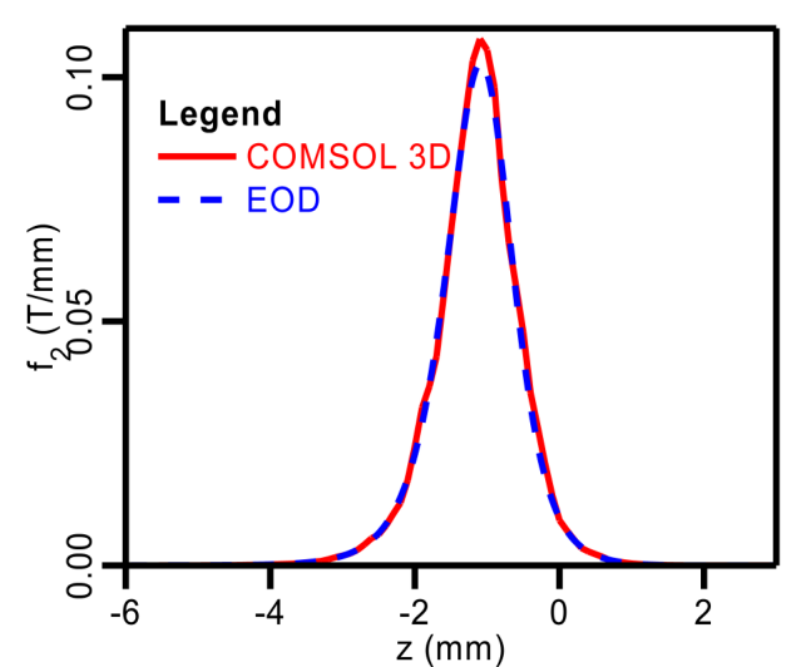

b)

Figure 5. Axial field functions scaled for a) shift and b) ellipticity $1 \mu \mathrm{m}$. Excitation of the lens 500 Ampere-turns.

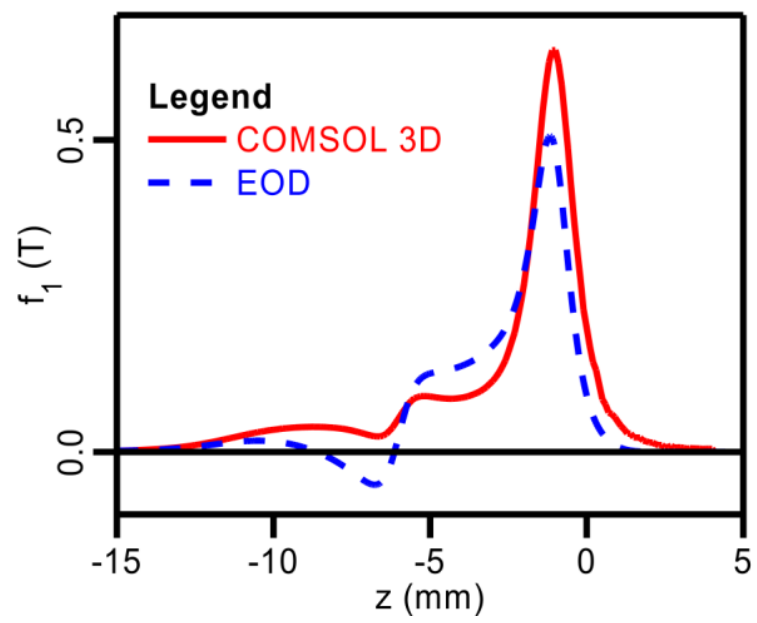

a)

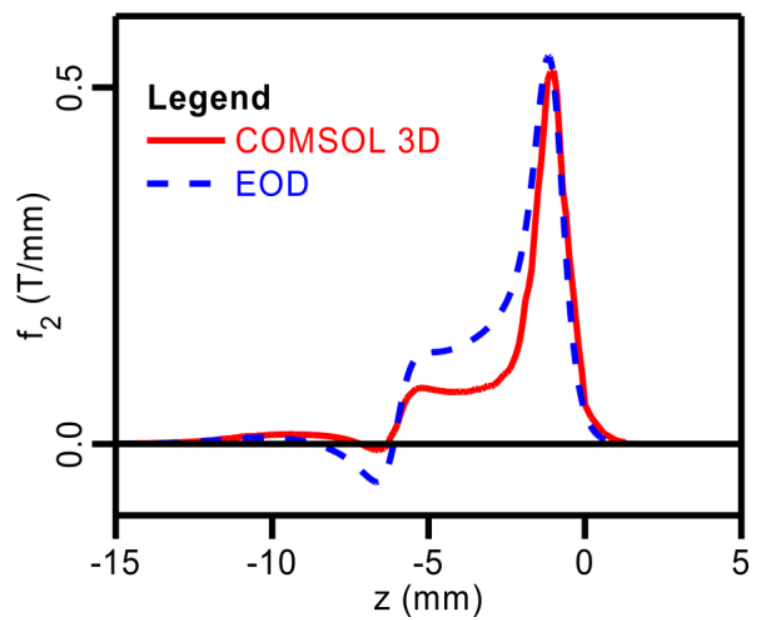

b)

Figure 6. Axial field functions scaled for a) shift and a) ellipticity $1 \mu \mathrm{m}$. Excitation of the lens 16000 Ampere-turns.

Obviously direct application of Sturrock's theory does not work for saturated lenses. The theory puts zero value of the perturbation potential on every surface that is not misaligned. The potential of the perturbation field is proportional to the radial component of the magnetic field intensity if the surfaces are parallel to the optical axis, or according to equations (1) and (2). The behavior of the perturbation field is therefore similar to the field of the radial coil used in Figure 3 where the zero boundary conditions of Sturrock's theory correspond to the linear case. The field of the radial coil in Figure 4 is strongly dependent on the material permeability and penetrates through the material in the nonlinear case. From the dependence of the field of deflector coils on the permeability of the magnetic material in Figure 1 it can be seen that a significant change of the axial field is for material relative permeability below 100. The validity of the Sturrock's theory can be therefore supposed only for the materials with higher value of relative permeability. 


\section{Conclusion}

The influence of saturated magnetic materials on fields of deflectors and parasitic fields was demonstrated on the saturated magnetic lens of Tsuno. The calculated field of toroidal and saddle deflection coils in the vicinity of magnetic material are in good agreement with published results. The significant change of the field of deflector next to magnetic material is observed if the material relative permeability falls below 100 . This value of the material relative permeability can be also considered as a limit for the applicability of the Sturrock's perturbation theory used to calculate parasitic fields caused by small shift, ellipticity and tilt of the pole piece. For the material relative permeability below 100 the zero perturbation potential for the unperturbed material surfaces prevents the penetration of parasitic fields through saturated material and the results of perturbation theory are no longer valid [11].

\section{References:}

[1] J Zlámal and B Lencová, Nucl. Instr. Meth. A 645 (2011), p. 278.

[2] B Lencová, Nucl. Instr. Meth. A 519 (2004), p. 133.

[3] M Lenc and B Lencová, Rev. Sci, Instrum. 68 (1997), p. 4409.

[4] B Lencová, M Lenc and K D van der Mast, J. Vac. Sci. Technol. B 7 (1989), p. 1846.

[5] O Sháněl, J Zlámal and M Oral, Ultramicroscopy 137 (2014), p. 1.

[6] M Kato and K Tsuno, IEEE Trans. Magn. 26 (1990), p. 1023.

[7] W Scherle, Berechnung von magnetischen Ablenksystemen, Dissertation, Tübingen 1983.

[8] PW Hawkes and E Kasper in "Principles of Electron Optics, Vol. 2", (Academic Press) 1996, p. 835.

[9] PA Sturrock, Phil. Trans. Roy. Soc. London A 243 (1951), p. 387.

[10] B Lencová, Optik 79 (1988), p. 1.

[11] This work was supported by European Regional Development Fund (CEITEC-

CZ.1.05/1.1.00/02.0068). 